\title{
Omics and organoids - a route to improved anti-venom
}

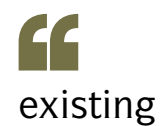

anti-venoms ... are limited by their variable efficacy and by adverse effects

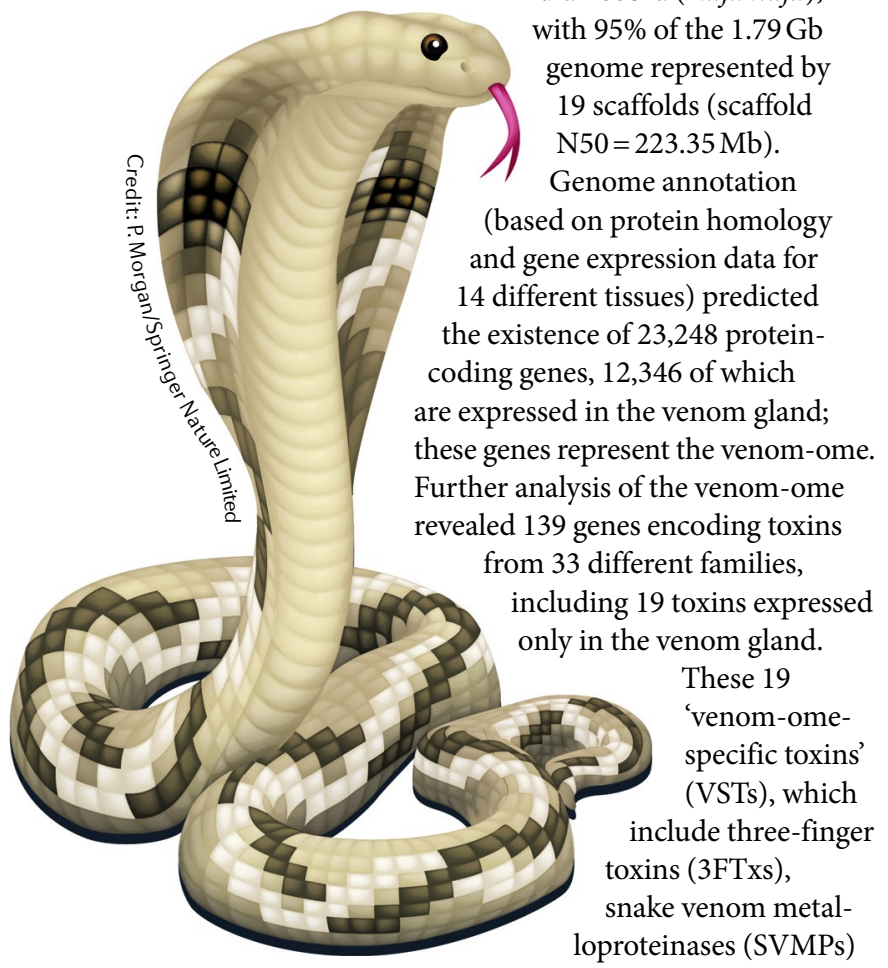

Each year, $\sim 5$ million people worldwide are affected by snake bites, $\sim 100,000$ fatally. However, the constituent toxins, toxinencoding genes and toxin-producing cells remain unknown or poorly characterized for most snake venoms, limiting the ability to produce humanized recombinant anti-venoms. Now, two independent studies report advances that potentially clear the way for better snake-bite treatments.

In a study published in Nature Genetics, Suryamohan et al. used a combination of short-read and long-read sequencing, optical mapping and chromatin interaction data to generate a high-quality de novo reference genome for the Indian cobra (Naja naja), with $95 \%$ of the $1.79 \mathrm{~Gb}$ $\mathrm{N} 50=223.35 \mathrm{Mb}$ ). Genome annotation the existence of 23,248 protein coding genes, 12,346 of which are expressed in the venom gland; these genes represent the venom-ome. Further analysis of the venom-ome ed 139 genes encoding toxins including 19 toxins expressed nom gland.

These 19 'venomific toxins' (VSTs), which include three-finger loproteinases (SVMPs) and cysteine-rich secretory venom proteins (CRISPs), likely represent the minimal core venom effector proteins. Together with other core effector proteins, they target multiple systems in the body and elicit cardiovascular, neural and muscular symptoms, among others.

The authors propose that cataloguing the complement of VSTs in the venom of $N$. naja and other snakes will provide a route to optimize existing anti-venoms, which are limited by their variable efficacy and by adverse effects. Furthermore, it will enable the development of new custom cocktails of activitytested, humanized anti-venoms using recombinant technologies.

In the second study, published in Cell, Post et al. derived and characterized snake venom gland organoids from adult stem cells. A generic 'expansion' cocktail for mammalian epithelial organoids was used to successfully derive organoids from dissociated venom glands for nine snake species (four viperids and five elapids, including Aspidelaps lubricus cowlesi). The organoids were capable of almost indefinite expansion under these conditions until differentiation was induced by switching to the 'differentiation' cocktail (that is, by withdrawing growth factors). The authors suggest that these culture conditions may be suitable for deriving organoids from other snake species and indeed from vertebrates more broadly.

In the absence of annotated genomes for the nine snake species, organoid gene expression was investigated by assembling a de novo transcriptome for A. l. cowlesi. Toxins were found to be the most abundant class of transcripts; 3FTxs were most abundant, but SVMPs, CRISPs and Kunitz-type inhibitors (KUNs) were also detected, and their relative abundances were similar between organoids and venom gland tissue. Further analyses confirmed that toxins were both present and functional in organoid protein extracts.

In venom glands, different toxins have spatially distinct expression patterns and are produced by morphologically distinct cell types, resulting in cellular and regional heterogeneity. Clustering analysis of single-cell RNA sequencing data and derivation of region-specific organoids confirmed that this heterogeneity was retained in organoids.

Thus, venom gland organoids are a genetically tractable model system for investigating the composition and production of venoms, and their ability to express recombinant toxins will facilitate anti-venom development.

The N. naja reference genome and venom-ome and venom gland organoids represent valuable community resources. Beyond their contributions to improved snakebite treatments, they will provide a fuller understanding of venom biology and could guide development of venom-based drugs.

Dorothy Clyde

ORIGINAL ARTICLES Suryamohan, K. et al. The Indian cobra reference genome and transcriptome enables comprehensive identification of venom toxins. Nat. Genet. 52, 106-117 (2020) | Post, Y. et al. Snake venom gland organoids. Cell 180, 233-247 (2020) 